\title{
PSO and GA designed Pareto of Fuzzy Controller in AC Motor Drive
}

\author{
Nasseer k. Bachache and Jinyu Wen \\ College of Electrical and Electronic Engineering, Huazhong University of Science \\ and Technology(HUST), Wuhan 430074, China \\ tech_n2008@yahoo.com
}

\begin{abstract}
In electric vehicles EV, can be implemented a controller has highest extend of battery's lifetime meet a superior dynamic speed behavior? This research offer multi-objective approaching planned by a Particle Swarm Optimization (PSO) complementary with Genetic Algorithm (GA). This hybrid evolutionary learning is utilized as an automated method to generate the optimal parameters of fuzzy logic controller (FLC) type Mamdani. The Pareto front characterizes the speed controller of an Induction Motor (IM). where the first function is the error between the actual speed and desired speed, and the other function is the energy dissipated from the electrical supply during (10 sec), in present work the multi-objective optimization of PSO and GA have been implemented separately using two M-file/MATLAB, and compacted the results to sketch the global Pareto, while the evaluation of "fitness functions" of the two computational algorithms have been determined using SIMULINK$M A T L A B$, the simulation has a completely mathematical model of induction motor IM, voltage source inverter VSI and FLC. The FLC have been implemented using RSLogix 5000. The empirical results demonstrate that the proposed method realized a limited disbursed energy as possible as better dynamic behavior of the IM speed along the Pareto front.
\end{abstract}

Keywords: Multi-objective Particle Swarm Optimization MOPSO, Fuzzy Logic Control FLC, Electrical Vehicles EVs, Induction Motor IM

\section{Introduction}

Electric vehicles EV are desperately needed to applications of variable speed with a limited power supply such as battery. This application should be work under a high quality controller. In recent years the ac drive has been one of the most important strategies in speed control due to a high reliability of induction motor IM and the developed of power electronic field. Ac drive is a widespread claim of poly phase inverters to adjusting speed motor. The typical inverter drives are voltage source inverter VSI produces Pulse Width Modulated PWM. The controller of this system must be designed for the specified performance. Furthermore, the systems are normally based on the mathematical model of Ac motor parameters stator flux orientation SFO [1]. This system is a highly nonlinear and difficult to obtain an exact mathematical model. However, Fuzzy Logic Control (FLC) is a powerful controller tool, even if the system is a non-linear or accurate mathematical model is unavailable. But, FLC suffers from the drawback of tuning its parameters (number of membership functions MFs type, formulating rules, etc.). The tuning of scaling factors for this parameter can be done either 
interactively method (trial and error) or human expert [2]. Therefore, the tunings of the FLC parameters are necessitated to an effective method for tuning. Nowadays, several new intelligent optimization techniques have been emerged, such as Genetic Algorithms (GA), Simulated Annealing (SA), Ant Colony Optimization (ACO) and Bacteria Foraging Optimization (BFO) among these nature-inspired strategies the Particle Swarm Optimization (PSO) algorithm is relatively novel [3], PSO has received great attention in a control system. In paper [4] the fuzzy controller parameters generated by PSO for an AC-Drive speed controller, In paper [5] designed AC-Drive system depends on the reference model (RF) on line with adaptive fuzzy controller in seven symmetrical triangular memberships for each inputs, in [6] is described an AC-Drive incorporates both the features of tracking and regulating in one Fuzzy Logic controller based on a "floating membership function", all these research didn't care to calculate the power dissipated or the losses. In this paper, we proposed a multi-objective function optimization for AC Drive under a powerful controller FLC to study how can get an optimized dynamic speed behavior with a limited power, Pareto diagram has two functions the first is reducing the error between the actual speed and desired motor speed while the other function is the energy dissipated from the electrical supply.

\section{Modeling of Three Phases I.M. and SPWM Inverter}

One of the most popular induction motor models derived from its equivalent circuit is Krause's model is based on a transformation the stator's currents and magnetic fluxes of the rotor to reference frame " $\mathrm{d}-\mathrm{q}$ " which rotates together with the rotor. The axis transformation is applied to transfer the three-phase parameters (voltage, current and flux) according to (d-q axes stationary frame). The most popular strategies in A.C drives should be used a Voltage Source Inverter (VSI) based on Sinusoidal Pulse Width Modulation (SPWM) [7]. In SPWM, the three output legs considered as three independent push-pull amplifiers. The reference frame model of IM and the invertor are illustrated in [4].

\section{Multi-objective Particle Swarm Optimization}

The multiple criteria nature of most real world problems has boosted investigation on multi-objective algorithms that can confront these problems effectively, with the smallest possible computational problem; Particle Swarm Optimization (PSO) has stimulated the researchers to solve a numerous single-objective optimization problems due to its simplicity, effectiveness and efficiency. PSO is a computation technique first proposed in 1995 by Kennedy and Eberhart [8, 9]. This method simulates a dynamic population behavior (fish swarm or bird flocks), It has been found to be a robust method in solving non-linearity or non-differentiability problems, PSO algorithm has been successfully applied in a wide of variety of optimization tasks in which it has shown a high convergence rate this algorithm explain briefly in [4] However, Multi-objective optimization is quite different from singleobjective optimization, the latter has one objective function so it is easy to calculate the global best position, then the basic PSO version is not effective to solve multi objective problem directly, because there are many objectives and incommensurable the global best particle among all these objectives, it is impossible to create all objective functions reach their 
minimum value at the same time [10]. The effective method to solve the desired solution thought MOPSO algorithm by converting Multiobjective problem into a Single - objective problem using Weighted Sum Approach (WSA), which represented in an equation (1)

$\min F(x)=\sum_{j=1}^{m} w_{j} \cdot f_{j}(k)$

Where: $w_{m} \in[0,1], \sum_{j=1}^{m} w_{m}=1$

$f_{j}(\mathbf{k})$ representing the best optimal finesses value of the $\mathrm{j}$-th objective function in the $\mathrm{k}$-th generation.

Hopefully by expression (1) we can get the Pareto solutions along 23 points against 23 Global best particles presented by the $\mathrm{w}_{1}=\left[\begin{array}{lllll}0.04 & 0.08 & 0.12 \ldots \ldots \ldots & 0.96\end{array}\right]$ and $\mathrm{w}_{2}=\left[\begin{array}{lll}0.96 & 0.92\end{array}\right.$ $0.88 \ldots \ldots . . .0 .04]$ making the current solutions to move toward the direction of the minimum distance from current position to each objective's best optimal value.

\section{Multi-objectives Genetic Algorithm (MOGA)}

Inspired by the natural evolution, John Holland in the early 1970s proposed the first basic principles of Genetic algorithm (GA), in recent years it was one of the most widely used tools for solving many optimization problems, relatively GA is a newly approach of optimum searching [11]. Through natural selection means the GA combines genes from two parent chromosomes to form two chromosomes (children) that have a high probability of having better fitness than their parents [12]. Mutation allows new areas of the response surface to be explored.

\section{MOPSO, MOGA Implemented with FLC}

Owning for one don't have experience of the system behavior, it is very difficult to forming the center and width of the triangle for the (inputs/output) Membership Fuzzy Functions (MFFs), in addition to get the optimized (MFFs) design. There is no formal framework for choice the parameters of FLC and hence the means of turning them and learning models in general have become an important subject of fuzzy control. The function of the fuzzy controller is to observe the pattern of the speed loop error signal and correspondingly updates the control signal, there are two input signals to the fuzzy controller: error (E) and change of error $(\mathrm{CE}), \mathrm{CE}$ means derivative of the error $(\mathrm{dE} / \mathrm{dt})$. A simple fuzzy logic controller of two inputs and one output can be designed; a seven triangle membership for each input and nine memberships for output. MOPSO can design positions of triangle memberships for input/output implementing by MATLAB/m-file program summarized in the flowchart shown in Figure 2; this figure illustrates the linked with MATLAB/SIMULINK the system simulation program by AC-drive model presented by (IM, inverter and FLC block sets) shown in Figure 1. The optimization criteria (Integrated of Time Weight Square Error ITSE) equation (2) is used to evaluate accuracy performance of the speed (FF1) to minimize the first function which evaluated in the variable (e2) exposed in Figure 1.

$$
\mathrm{FF} 1=\operatorname{ITSE}=\int_{0}^{\mathrm{t}} \mathrm{t} * \mathrm{e}^{2}(\mathrm{t}) \mathrm{dt}
$$


But, the optimization of the other function FF2 is evaluated by the variable (e) expressed the energy delivered to the motor; assessed by the integration of the power input (multiplying the voltage and the current). A set of good controller parameters can yield a good step response along the Pareto front that will result, and the same method of calculating fitness function we can sketch the Pareto of MOGA by Graphic User Interface (GUI) in the MATLAB program by the function optimtool ('gamultiobj').

By the two algorithms (MOPSO, MOGA) the dominated points can be determined along the global Pareto which presented by twenty one points. Each point has a controller consist two inputs one output. These controllers can compacted in one controller has three inputs and one output. The third input represents the desired energy according the life battery Figure 3 shows the flow chart of this work.

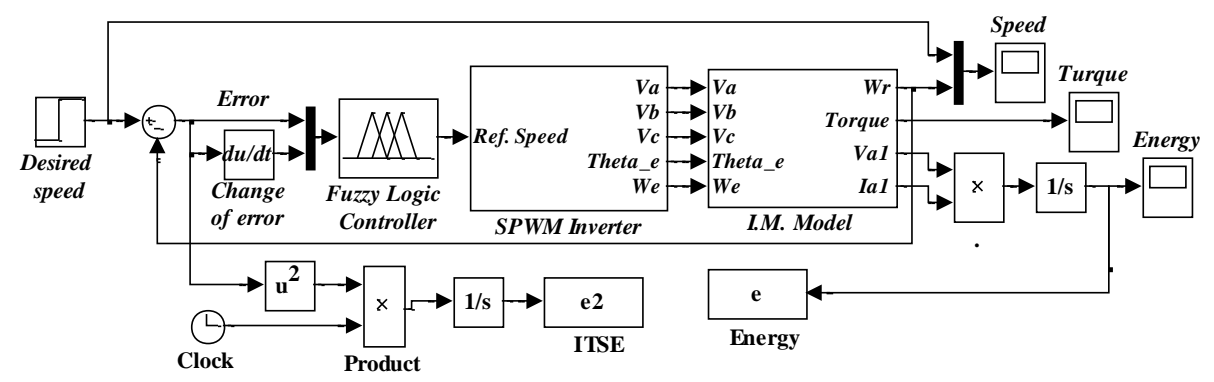

Figure 1. Simulation of AC-derive with FLC

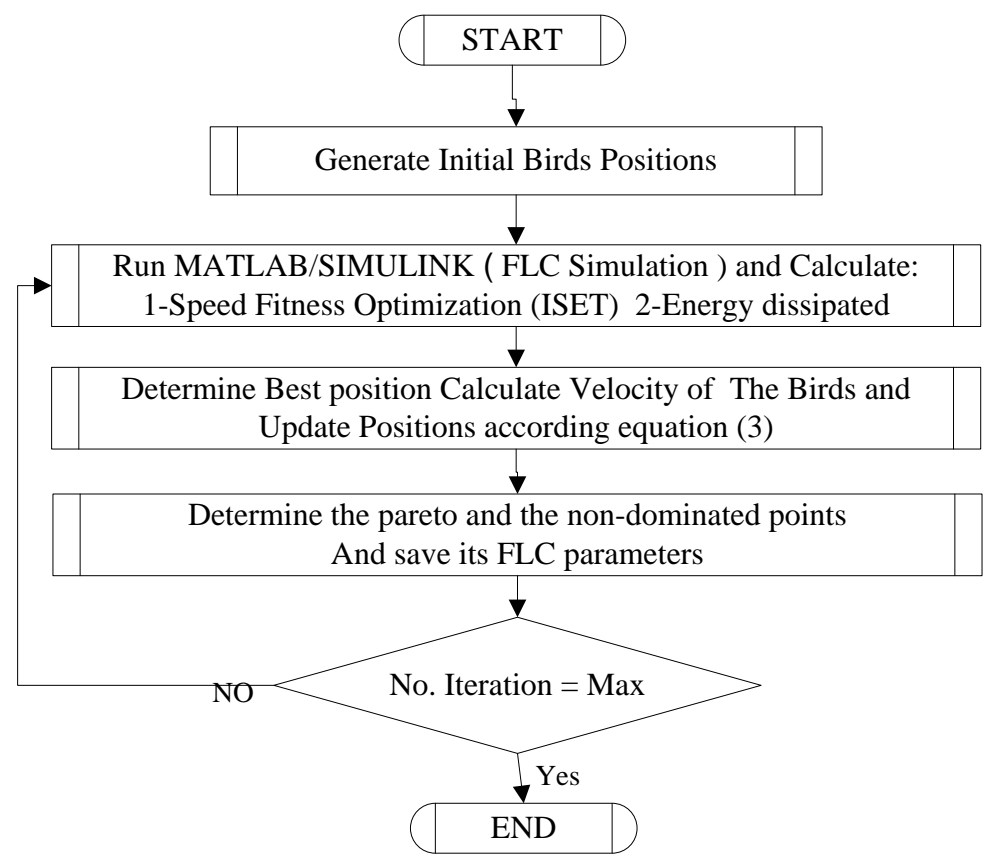

Figure 2. Flow chart of multi-objective PSO 


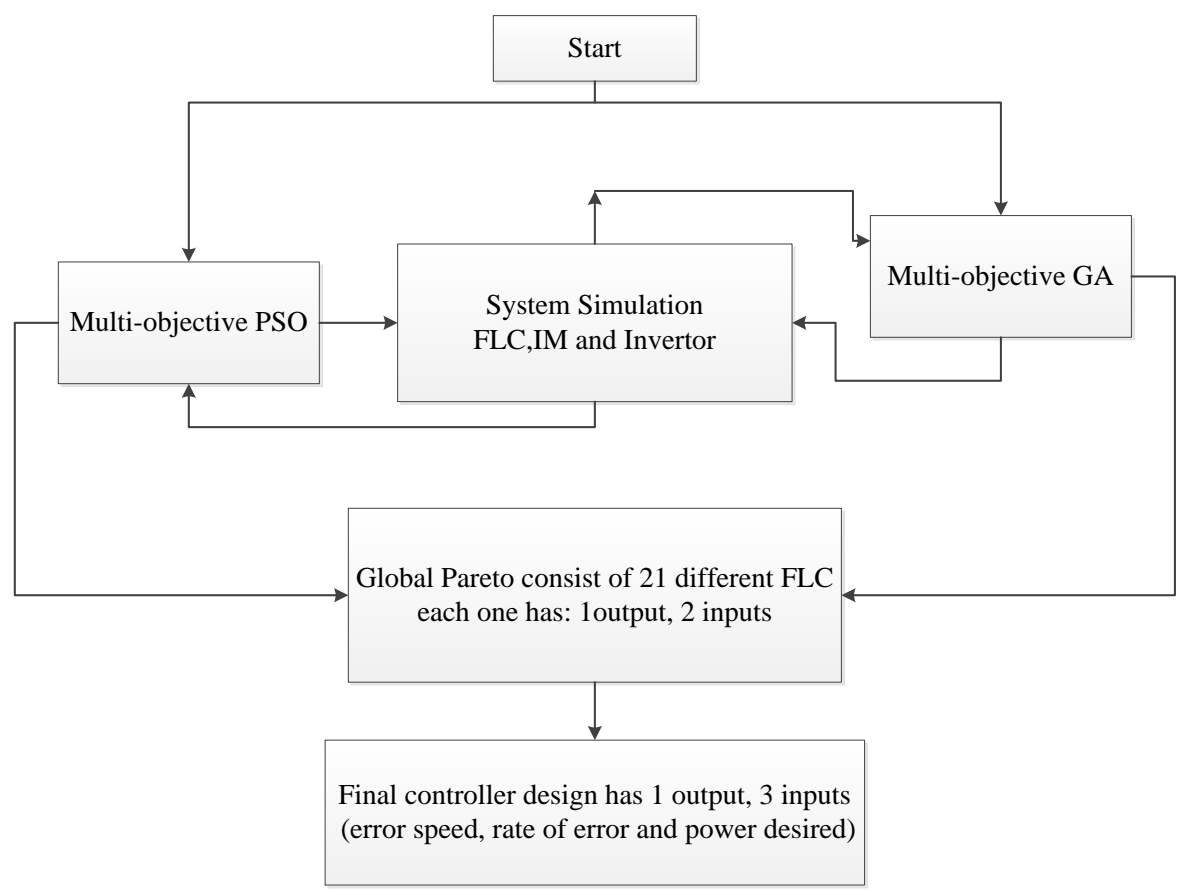

Figure 3. Flowchart illustrate PSO algorithm

\section{Programmable logic controller PLC implementation.}

Programmable Logic Controller (PLC) is a device used to solve complex control tasks in automation of electromechanical processes. The key contribution of this paper in field of PAWT is to design optimal FLC system by MATLAB and convert fuzzy system into PLC's fuzzy structure. Rockwell Software RSLogix5000 is used to program the PLC of Allen-Bradley Control Logix. The FuzzyDesigner feature within RSLogix 5000 software works as an editor to use own formed FLC in the Logix5000 family of controllers. Typically, the proposed controller doesn't create an add-on instruction in FuzzyDesigner software. But, we import the controller form MATLAB throw Ole Process Control (OPC) using opctool.

\section{Simulation Result}

Figure 4 shows the space of operating points (dominated, non-dominated) and determined the Pareto curve of the multi objective optimization of the two functions the ITSE of the difference between the desired speed and the actual speed on the $\mathrm{x}$ axis and the other function on y-axis is the energy dissipated, the figure shows the lowest ITSE (ITSE =1. 322) this controller spend the largest scale of energy (49.93). Figure 5 shows the comparison between the two Pareto obtained by MOPSO and MOGA. Figure 6 shows proposed Multi objective optimization matching MOPSO and MOGA. Figure 7 shows the system step response of the two controllers designed by the proposed method which one need large energy with better dynamic behavior and the other Lower Dissipated of Energy but it has the worst dynamic 
speed behavior. Figure 8 show the energy dissipated of the two different controllers. Figures 9 and figure10 show two deferent FLC memberships in inputs/output designed by MOPSO.

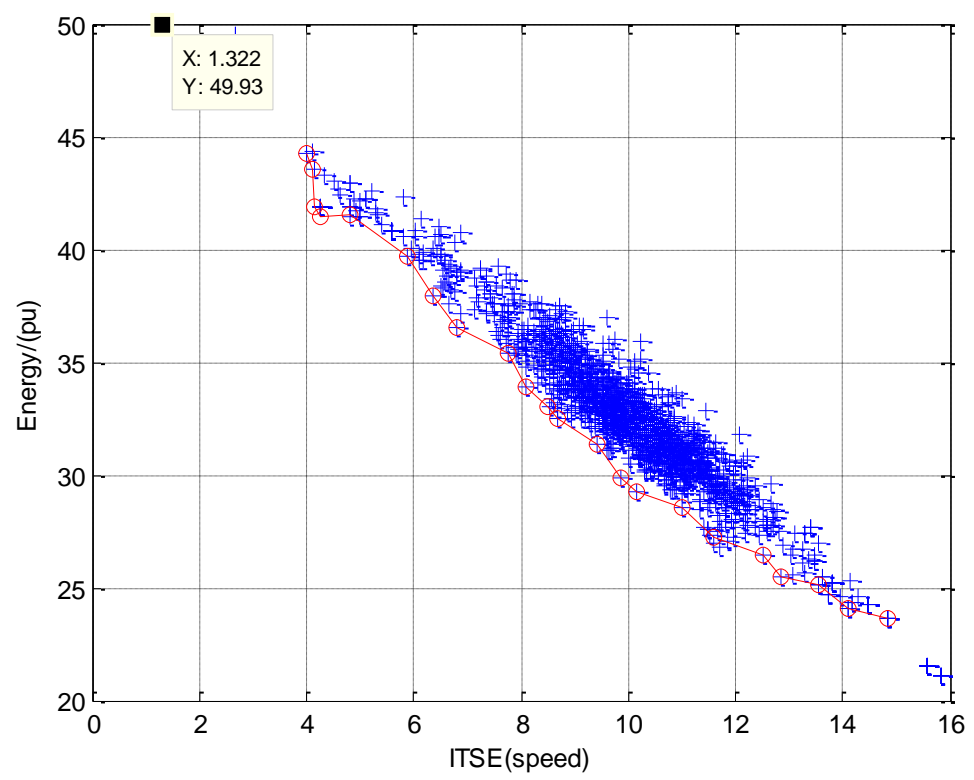

Figure 4. Pareto of MOPSO and the all dominated points

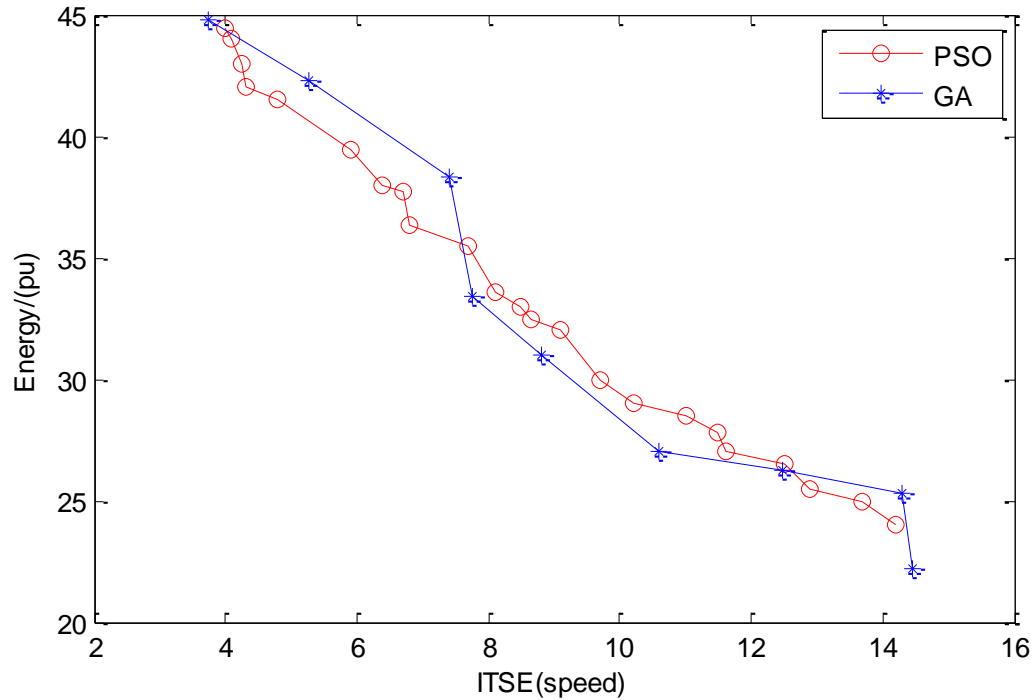

Figure 5. Comparison of Pareto by MOPSO and Pareto by MOGA 


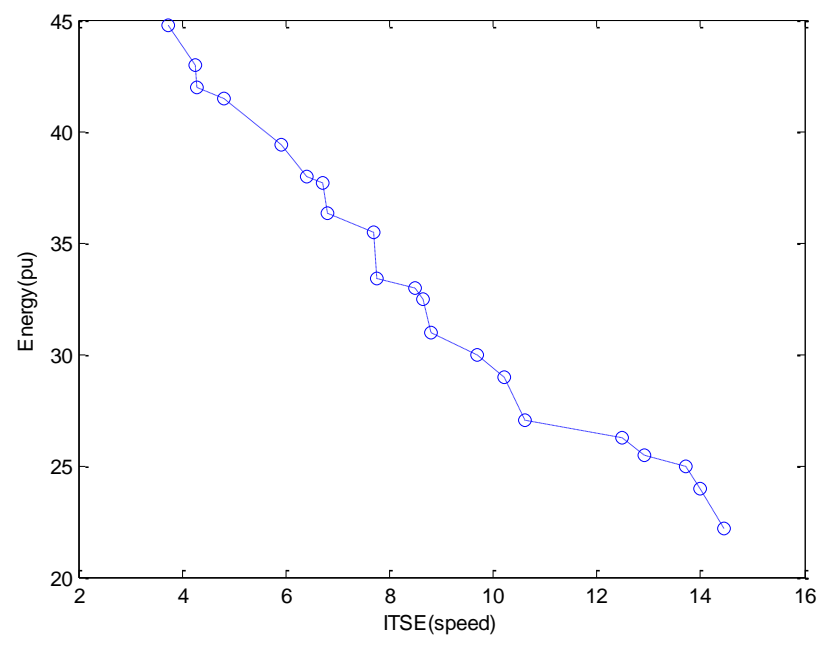

Figure 6. Proposed Pareto designed by MOPSO and MOGA

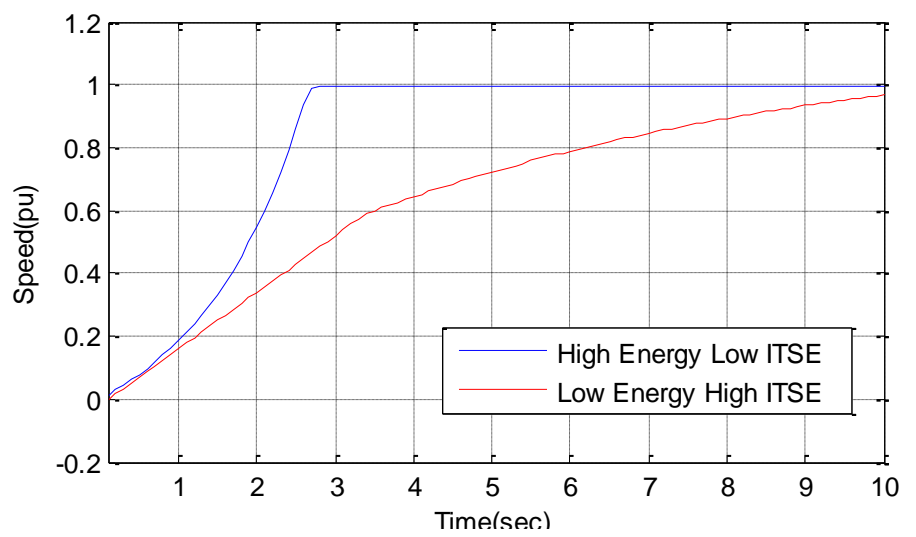

Figure 7. Step response of speed for two FLC

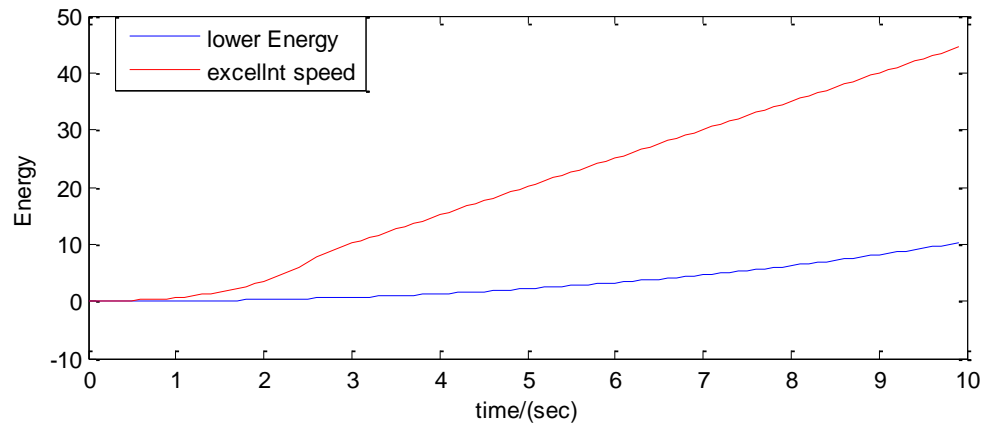

Figure 8. Energy disputed Step response for two FLC 


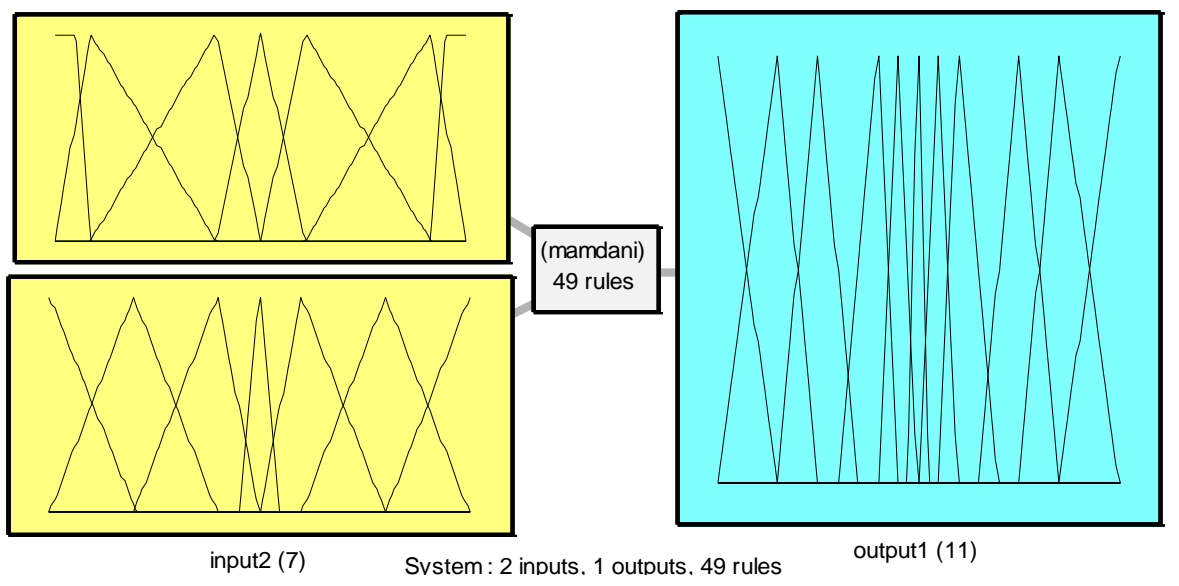

Figure 9. FLC designed by MOPSO for excellent speed dynamic behavior

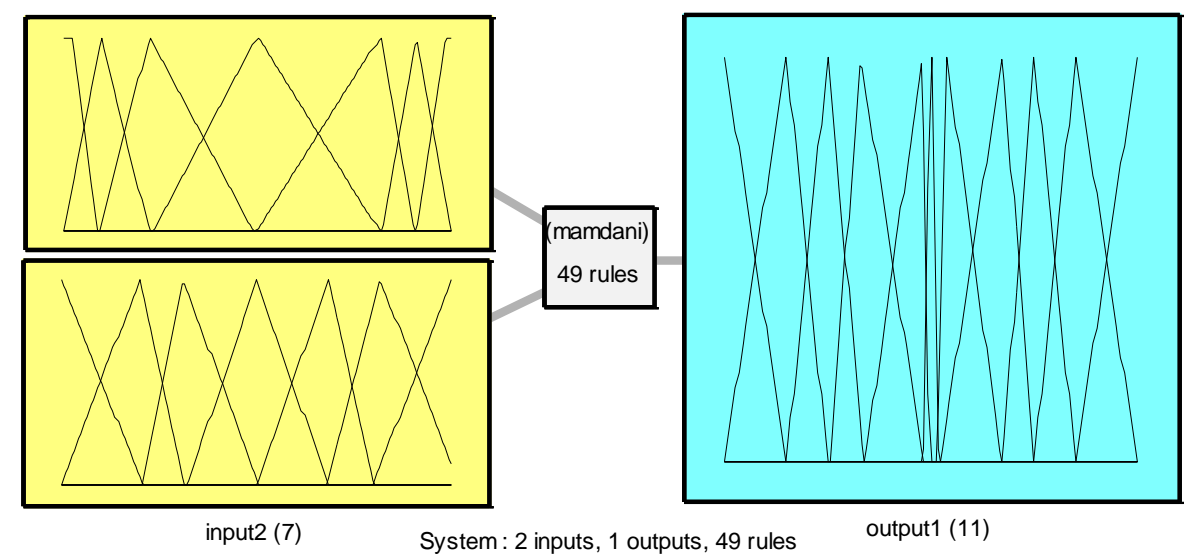

Figure 10. FLC designed by MOGA for lower energy dissipated

\section{Conclusions}

In this paper, we have proposed MOPSO, which is an evolutionary multi-objective algorithm developed to generate a set of FLCs with different function's accuracy of dynamic speed and energy, which is measure a numerous of membership (MF) antecedent conditions. The proposed method is able to learn the MF parameters. This allows us to take the better controller during the life of the battery or just before it will be discharged. A multi-objective PSO algorithm MOPSO can solve the engineering constrained optimization problem by converting into a single objective problem using Weighted Sum Approach (WSA) so it is easier to implement, and give the result sometime better than Genetic Algorithm (MOGA) and MOPSO algorithm is able to find competitive solutions and converge quickly. The simulation test results validate that the fuzzy logic controller designed by (MOPSO) can be successfully cooperated with (MOGA) to improve the global Pareto. The controller of AC drive should be designed according the specific motor efficiency. During steady states and 
transient the optimal value of the favorable case can be minimized in order to achieve less power consumption and to reduce the convergence time.

\section{References}

[1] M. Wlas, H. Abu-Rub and J. Holtz, "Speed Sensorless Nonlinear Control of Induction Motor In The Field Weakening Region", Proceedings of the IEEE 13th International Power Electronics and Motion Control Conference, (2008), pp. 1084-1089.

[2] M. Dubey, "Design of Genetic Algorithm Based Fuzzy Logic Power System Stabilizers in Multimachine Power System”, Proceedings of IEEE POWERCON \& Power Conference, (2008) October 12-15; New Delhi, India.

[3] M. R. Rapaic, Z. Kanovic and Z. D. Jelicic, "A Theoretical and Empirical Analysis of Convergence Related Particle Swarm Optimization”, Proceedings of WSEAS Transactions on Systems and Control, vol. 11, no. 4, (2009) November.

[4] N. K. Bachache and J. Wen, "Particle Swarm Optimize Fuzzy Logic Memberships of AC-Drive", Springer, LNCS, vol. 3771, (2012), pp. 460-459.

[5] Y. Kung, M. Wang and C. Huang, "Digital Hardware Implementation of Adaptive Fuzzy Controller for AC Motor Drive", Proceedings of the 33rd Annual Conference of the IEEE Industrial Electronics Society (IECON), (2007), Taipei, Taiwan.

[6] A. G. Keskar and K. L. Asanare, "Floating Membership Fuzzy Logic Controller for Adaptive Control of AC Drive", IEEE Catalog Number, (1997), Guhariies, Portugal.

[7] O. A. Lara, J. Ekanayake, P. Cartwright and M. Hughes, "Wind Energy Generation Modeling and Control", A John Wiley and Sons, Ltd., (2009).

[8] A. Chen, G. Yang and Z. Wu, "Hybrid discrete particle swarm optimization algorithm for capacitated vehicle routing problem”, Journal of Zhejiang University SCIENCE, (2006), pp. 607-614.

[9] C. Zhou, L. Gao, H. Gao and K. Zan, "Particle Swarm Optimization for Simultaneous Optimization of Design and Machining Tolerances", Springer-Verlag Berlin Heidelberg, (2006), pp. 873-880.

[10] D. Tan, W. Luo and Q. Liu, "Multi-objective particle swarm optimization algorithm for engineering constrained optimization problems", Proceedings of IEEE International Conference Granular Computing GRC'09, (2009), pp. 523-528.

[11] F. Martinez and M. Gastiblanco, "Increase the Boost Converter Performance Using Genetic Algorithms", Journal on Electronics and Electrical Engineering, vol. 2, no. 1, (2008).

[12] L. Davis, "A Handbook of Genetic Algorithm", Van Nostrand Reinhold, New York, (1990). 
International Journal of Control and Automation Vol.6, No.5 (2013) 